\title{
Strength of Knowledge Assessment for Risk Informed Decision Making
}

\author{
Tasneem Bani-Mustafa ${ }^{(1)}$, Zhiguo Zeng ${ }^{(1)}$, Enrico Zio ${ }^{(1),(2)}$, Dominique Vasseur ${ }^{(3)}$ \\ (1) Chair on System Science and the Energetic Challenge, EDF Foundation
}

Laboratoire Genie Industriel, CentraleSupélec, Université Paris-Saclay,

3 Rue Joliot Curie, 91190 Gif-sur-Yvette, France

(2) Energy Department, Politecnico di Milano, Via La Mase 34, Milan, 20156, Italy

(3) EDF R\&D, PERICLES (Performance et prévention des Risques Industriels du parc par la simulation et les Etudes)

EDF Lab Paris Saclay - 7 Bd Gaspard Monge, 91120 Palaiseau, France

\begin{abstract}
Risk Informed Decision Making (RIDM) is based on risk metrics obtained from a Probabilistic Risk Assessment (PRA). For plants exposed to multiple hazards, Multi-Hazards Risk Aggregation (MHRA) is necessary to inform decisions. In practice, this is often done by a simple arithmetic summation over the different risk contributors, without taking into account that the state of knowledge of the risk models of the different hazards can be quite different.

In this paper, we provide a hierarchical framework to assess the strength of knowledge that PRA models are based upon. The framework is organized in three attributes characterizing the knowledge which a PRA model is based upon (assumptions, data, phenomenological understanding). These attributes are further broken down into sub-attributes and, finally, "leaf" attributes that can be evaluated. The PRA models of two hazards groups for Nuclear Power Plants (NPPs) are considered and the strength of knowledge behind each model is assessed using the developed framework.
\end{abstract}

Keywords: Strength of knowledge, Probabilistic Risk Assessment (PRA), Risk Informed Decision Making (RIDM), MultiHazards Risk Aggregation (MHRA)

\section{Introduction}

In risk assessment, quantities are calculated to describe the magnitude and likelihood of the consequences from accidents that may develop from known hazards [1]. The confidence on the calculated risk indexes depends on the knowledge available to support the risk assessment [3-5]. For example, in the risk assessment of Nuclear Power Plants (NPPs), there is more experience and knowledge on internal events than other hazard groups like external flooding [1]. Evaluating the strength of knowledge of a risk assessment, is, then, important to evaluate how much confidence we can put on the risk outcomes, that are, then, used to inform decision making [2].

Research efforts have been conducted, recently, for linking knowledge, knowledge evaluation and knowledge management to Risk-Informed Decision-Making (RIDM) [4-7]. For example, in the nuclear industry, knowledge management has been identified as a key factor in sustaining nuclear power programs and maintaining their safety and security [3]. However, most of the existing works are qualitative in nature. A semi-quantitative method for evaluating the strength of knowledge has been proposed by Flage and Aven [4], where the strength of knowledge is evaluated in terms of four attributes: (i) phenomenological understanding and availability of trustable predicting models; (ii) reasonability and realism of assumptions; (iii) availability of reliable and relevant data, and information; (iv) agreement/disagreement among peers. The four attributes were assessed in three levels (minor, moderate and significant) and aggregated for strength of knowledge assessment [4]. Although the knowledge attributes proposed are plausible and reasonably complete, their definitions remain ambiguous. In addition, the evaluation of these attributes is somewhat intangible in practice, since it is done by simple scoring based on a plain description of the attributes. To overcome this problem, we expand the work in [5] and introduce a hierarchical tree-based framework for evaluating the state of knowledge.

The rest of the paper is organized as follows. In Sect. 2, we present the developed framework for strength of knowledge assessment. Section 3 applies it on a case study of two hazard groups considered in NPPs risk assessment. Finally, in Sect 4, the paper is concluded with a discussion on potential future developments.

\section{Assessment framework}

We consider the strength of knowledge assessment of event tree models which are widely applied in PRA of NPPs. The events probabilities in the event tree model might are typically calculated by fault tree models. The risk index associated to a given consequence (e.g. the probability of core damage) is calculated by summing the values of the risk index from several risk models:

$$
R=\sum_{i=1}^{n_{O}} \sum_{j=1}^{n_{S, i}} R_{i, j}
$$

where $n_{O}$ is the number of operation states $(\mathrm{O}), n_{S, i}$ is the number of accident sequences (scenarios, $S$ ) that in operation state $i$ can lead to the given consequence. Each $R_{i, j}$ in (1) quantifies the specific risk index under scenario $j$ (e.g., medium flood level) in operation state $i$ (e.g., emergency shutdown).

The risk models used to calculate the risk index $R_{i, j}$ values are characterized by initial events (IEs), basic events (BEs) and the combinations of the latter into minimal cut sets (MCSs). In practice, it can often be assumed that the MCSs are mutually exclusive; then, $R_{i, j}$ can be calculated by [5]:

$$
R_{i, j}=\sum_{k=1}^{n_{M C S, i, j}} \prod_{q=1}^{n_{B E, k}} P_{B E, q}
$$

where $n_{M C S, i, j}$ is the number of minimal cut sets in the risk model for operation state $i$ and scenario $j, n_{B E, k}$ is the number of basic events in the $k$ th minimal cut set, and $P_{B E, q}$ is the probability of having the $q$ th basic event. The five elements, $S$, $\mathrm{O}, \mathrm{IE}, \mathrm{BE}$ and MCS, fully define a PRA model, as shown in 
Figure 1. In this paper, we refer to these five elements as "atomic elements".

To assess the strength of knowledge of a PRA model, all the five atomic elements need to be considered. In practice, however, PRA models are very complex and contain many scenarios and

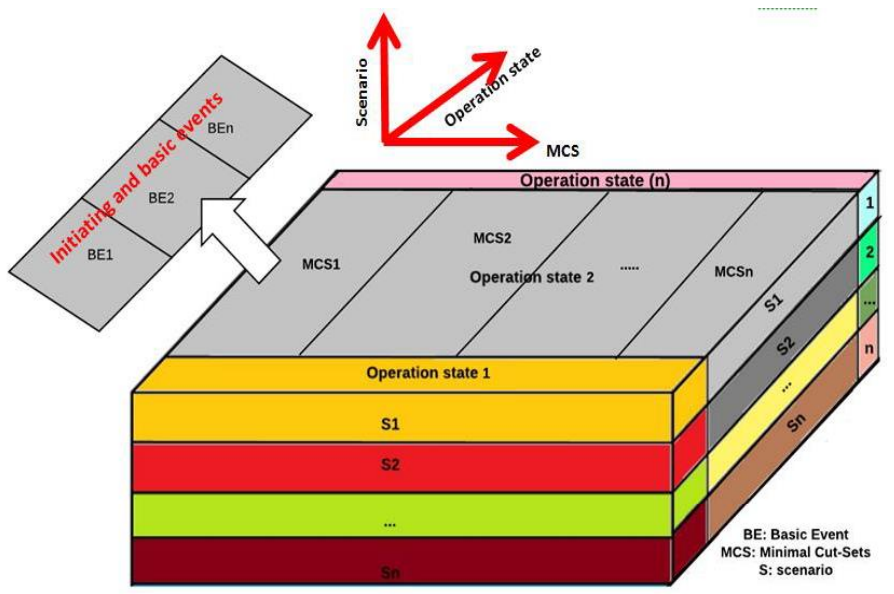

Figure 1 Atomic elements of a PRA model

operation states combined in large and complex fault trees and event trees, that consist of thousands of BEs and MCSs [6]. For such a complex risk assessment model, it is not practical to consider all atomic elements for evaluating the strength of knowledge. To address this problem, in this work, we first develop a reduced-order model for (1), in order to limit the number of atomic elements that need to be analyzed.

A flowchart of the developed knowledge assessment method is given in Figure 2. The first step involves developing a reducedorder model for the original risk assessment model. A detailed discussion on how to construct the reduced-order model is given in Sect. 2.1. Then, the strength of knowledge supporting each atomic element in the reduced-order model is assessed by an Analytical Hierarchy Process (AHP), as illustrated in Sect. 2.2. Finally, the strength of knowledge of each element is aggregated to evaluate the strength of knowledge of the entire PRA model. A detailed discussion is given in Sect. 2.3.

Reduced-order PRA model construction

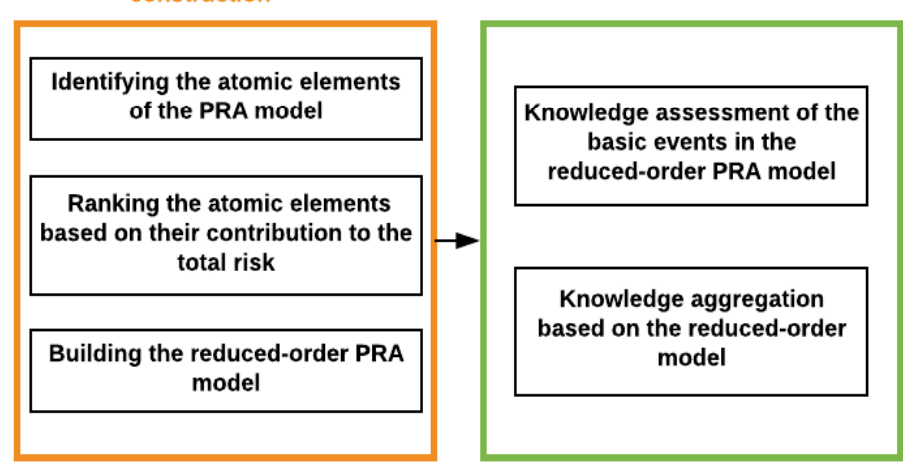

Figure 2 Steps of PRA model knowledge assessment

\subsection{Reduced-order PRA model construction}

It is often observed in PRA models that most of the contribution to the total risk is due to a small number of elements of the problem (known as "Pareto principle") [7]. We can, then, reduce the PRA model into a reduced-ordered model, which consists of only the most important "elements".
The procedure for constructing the reduced-order model comprises of three steps. Firstly, the number of operation states $n_{O}$ is reduced to $n_{O, R e d}$, as follows:

- Calculate the risk $R_{O_{i}}$ for each operation state:

$$
R_{O_{i}}=\sum_{j=1}^{n_{S, i}} R_{i, j}, \quad 1 \leq i \leq n_{O}
$$

where $R_{i, j}$ is calculated by (2).

- $\quad$ Rank $R_{O_{i}}$ in descending order.

- Find the minimal $n_{O, R e d}$, so that

$$
\frac{\sum_{i=1}^{n_{O, R e d}} R_{O_{i}}}{R} \geq \alpha
$$

where $\alpha$ is the fraction of total risk that can be reproduced by the operation states in the reduced-order model (in the case study in Sect. 3.2.1, we assume that $\alpha=0.8)$.

- Keep only operation states for $i=1, \cdots, n_{O, \text { Red }}$; operation states with $i>n_{O, R e d}$ are eliminated.

The second step is to define the reduced number of scenarios $n_{S, R e d, i}$ for each operating state $i$ in the reduced-order model, where $i=1, \cdots, n_{O, R e d}$ :

- $\quad$ For $i=1, \cdots, n_{O, R e d}$, calculate the risk $R_{i, j}, 1 \leq j \leq$ $n_{S, i}$ by (2).

- $\quad$ Rank $R_{i, j}$ in descending order.

- Find the minimal $n_{S, R e d, i}$ so that,

$$
\frac{\sum_{j=1}^{n_{S, R e d, i}} R_{i, j}}{R_{O, i}} \geq \beta
$$

where $R_{O_{i}}$ is calculated by (3) and $\beta$ is the fraction of total risk that can be reproduced by the scenarios in the reduced-order model (in the case study in Sect. 3.2.1, we assume that $\beta=0.8$ ).

- $\quad$ Keep only scenarios for $j=1, \cdots, n_{S, R e d, i}$; scenarios with $j>n_{S, R e d, i}$ are eliminated.

Finally, the number of minimal cut sets $n_{M C S, i, j}$ is tailored to $n_{M C S, R e d, i, j}, i=1, \cdots, n_{O, R e d}, j=1, \cdots, n_{S, \text { Red }, i}$ :

- Calculate $R_{i, j, k}$ by:

$$
R_{i, j, k}=\prod_{q \in M C S_{i, j, k}} P_{B E, q}, \begin{gathered}
1 \leq i \leq n_{O, R e d} \\
1 \leq j \leq n_{S, R e d, i} \\
1 \leq k \leq n_{M C S, i, j}
\end{gathered}
$$

- $\quad$ Rank $R_{i, j, k}$ in descending order.

- $\quad$ Find the minimal $n_{M C S, R e d, i, j}$ so that,

$$
\frac{\sum_{k=1}^{n_{M C S, R e d, i, j}} R_{i, j, k}}{R_{i, j}} \geq \gamma
$$

where $R_{i, j, k}$ is calculated by (6) and $\gamma$ is the fraction of total risk that can be reproduced by the minimal cut sets in the reduced-order model (in the case study in Sect. 3.2.1, we assume that $\gamma=0.8$ ).

- $\quad$ Keep only minimal cut sets for $k=1, \cdots, n_{M C S, R e d, i, j}$; minimal cut sets with $k>n_{M C S, R e d, i, j}$ are eliminated.

Assuming that the MCSs are mutually exclusive, the total risk of the reduced-order PRA model can be calculated by:

$$
R_{R e d}=\sum_{i=1}^{n_{O, R e d}} \sum_{j=1}^{n_{S, R e d, i}} \sum_{k=1}^{n_{M C S, R e d, i, j}} \prod_{q \in M C S_{i, j, k}} P_{B E, q}
$$

Note that from (4), (5) and (7), the reduced order risk $R_{R e c}$ can reconstruct $\alpha \times \beta \times \gamma$ of the total risk $R$. Only the events that are contained in the reduced-order model (8) are used for assessing the strength of knowledge of the PSA. 


\subsection{Knowledge assessment for the risk elements}

Once the reduced-order model is constructed, the strength of knowledge of each atomic element in such model is evaluated. In Section 2.2.1, we present a tree-based hierarchical framework for knowledge assessment. Then, in Section 2.2.2, we show how to proceed with the evaluation using the Analytical Hierarchy Process (AHP) method.

\subsubsection{Knowledge assessment framework}

A tree-based hierarchical framework is here developed for knowledge assessment, as shown in Figure 3. The strength of knowledge, represented by $K$ (Level 1$)$, represents the solidity of knowledge that supports a PRA model. A higher value of strength of knowledge indicates that the PRA model is supported by trustable evidence and reliable knowledge, and, therefore, its results can be taken with confidence.

As in Flage and Aven [4], we evaluate the strength of knowledge in terms of three attributes: assumptions $\left(K_{1}\right)$, data $\left(K_{2}\right)$ and phenomenological understanding $\left(K_{3}\right)$. The attribute $K_{1}$ represents the adequacy, solidity and plausibility of the assumptions upon which the model is based; $K_{2}$ represents the amount and quality of the available data that are used to estimate the parameters of the model; $K_{3}$ represents the knowledge behind the phenomenon described in the model.
For their evaluation, the three attributes are further decomposed into sub-attributes. In particular, assumptions $\left(K_{1}\right)$ is evaluated in terms of quality of assumptions $\left(K_{11}\right)$, value ladenness $\left(K_{12}\right)$ and impact $\left(K_{13}\right)$; data $\left(K_{2}\right)$ is evaluated in terms of the amount of data $\left(K_{21}\right)$ and the reliability and consistency of data $\left(K_{22}\right)$; phenomenological understanding $\left(K_{3}\right)$ is evaluated in terms of years of experience of the experts involved in the model development $\left(K_{31}\right)$, number of experts involved $\left(K_{32}\right)$, academic evidence $\left(K_{33}\right)$ and industrial evidence $\left(K_{34}\right)$. Value ladenness and reliability and consistency of data are further decomposed into "leaf" sub-attributes in level 4 for their evaluation, as shown in Figure 3.

The tree structure in Figure 3 is constructed based on a thorough literature review related to trustworthiness and validity assessment of PRA/QRA. References related to the construction of the tree model are given in Table 1. It should be noted that for phenomenological understanding, few references directly consider its assessment. A comprehensive understanding of phenomena requires its explanation [8], which depends on the capability of the experts involved in the risk modeling and analyses. Then, four sub-attributes are proposed for the assessment of phenomenological understanding: (i) industrial evidence; (ii) academic evidence; (iii) number of experts involved; (iv) number of years of experience in the domain).

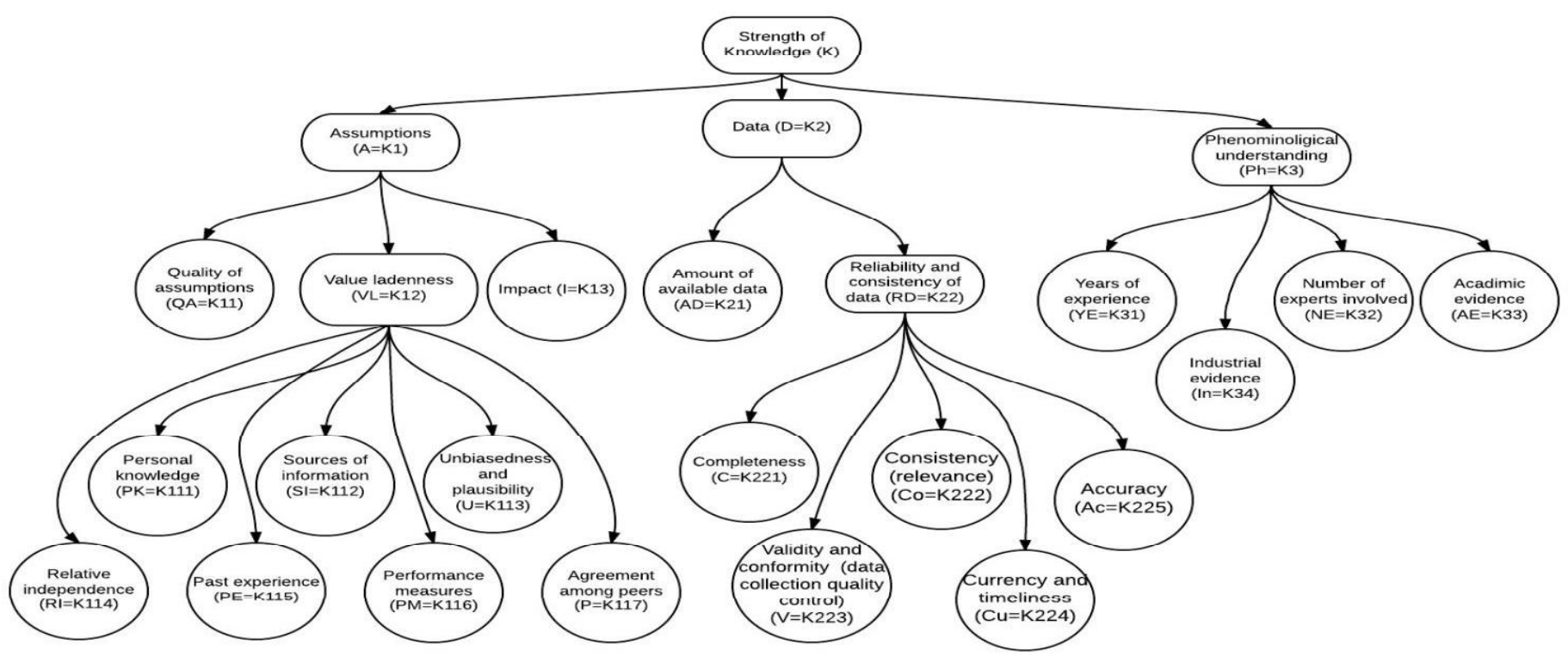

Figure 3 A hierarchical tree-based framework for knowledge assessment

Table 1. References that justify the model in Figure 3

\begin{tabular}{|l|l|}
\hline Attributes & References \\
\hline Strength of knowledge is evaluated by $K_{1}, K_{2}$ and $K_{3}$. & {$[4]$} \\
\hline $\begin{array}{l}\text { Realism and plausibility of assumptions }\left(K_{1}\right) \text { is evaluated by the quality of assumptions }\left(\mathrm{K}_{11}\right), \text { the value } \\
\text { ladenness and subjectivity of the experts }\left(\mathrm{K}_{12}\right) \text { and sensitivity analysis on the assumptions }\left(\mathrm{K}_{13}\right) .\end{array}$ & {$[9] ;[10] ;[11]$} \\
\hline Value ladenness $\left(K_{2}\right)$ is defined by $K_{111}-K_{117}$. & $\begin{array}{l}10] ;[9] ;[12] ; \\
{[13] ;[14]}\end{array}$ \\
\hline Data $\left(K_{2}\right)$ is evaluated in terms of amount of available data $\left(K_{21}\right)$ and reliability of data $\left(K_{22}\right)$. & {$[4]$} \\
\hline Reliability of data is defined by: (i) completeness; (ii) consistency; (iii) accuracy; (iv) validity; (v) timeliness. & {$[15] ;[16] ;[17]$} \\
\hline
\end{tabular}

\subsubsection{Evaluation using AHP}

Given the hierarchical tree in Figure 3, the assessment of the strength of knowledge is carried out within a multi-criteria decision analysis (MCDA) framework. AHP is adopted [18], as it is fit for both quantitative and qualitative evaluation of attributes and factors [19] and for group decision making [20].
A first step in applying AHP is to evaluate the "leaf" attributes (the non-decomposable attributes in Figure 3). A score between 1 and 5 is used to represent the strength of knowledge with respect to each "leaf" attribute, where 1 represents the lowest knowledge level and 5 represents the highest knowledge level. The score is evaluated based on some predefined evaluation 
criteria. Due to page limits, we only present the evaluation criteria for $K_{11}$ as an example (See Table 2).

Table 2. Quality of assumptions scoring guidelines

\begin{tabular}{|c|c|c|c|}
\hline $\begin{array}{l}\text { Score } \\
\text { Attribute }\end{array}$ & 1 & 3 & 5 \\
\hline 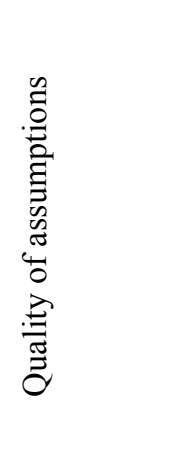 & $\begin{array}{l}\text { The } \\
\text { assumptions } \\
\text { are based on } \\
\text { weak } \\
\text { knowledge } \\
\text { and not } \\
\text { realistic } \\
\text { (conservative } \\
\text { assumptions } \\
\text { or over- } \\
\text { optimistic) }\end{array}$ & $\begin{array}{l}\text { The } \\
\text { assumptions } \\
\text { are } \\
\text { acceptable } \\
\text { based on } \\
\text { moderate } \\
\text { knowledge, } \\
\text { simple } \\
\text { model and } \\
\text { extrapolated } \\
\text { data }\end{array}$ & $\begin{array}{l}\text { The } \\
\text { assumptions } \\
\text { are based on } \\
\text { strong } \\
\text { knowledge } \\
\text { and } \\
\text { established } \\
\text { theory, } \\
\text { verified by } \\
\text { peer review } \\
\text { and very } \\
\text { plausible }\end{array}$ \\
\hline
\end{tabular}

Then, the inter-level priorities (weights) are determined for each attribute, sub-attribute and "leaf" attribute, denoted by $W\left(K_{i}\right)$, $W\left(K_{i j}\right)$ and $W\left(K_{i j k}\right)$, respectively. Based on [14] and [20], a scale of 1-9 is used for evaluating the importance of each of these attributes relative to each other, with reference to their contribution to the parent attribute: a value of 1 is assigned when two attributes of the same level of the hierarchy are equally important and 9 is assigned when one attribute is significantly more important than the other.

The strength of knowledge of the $i$ th atomic element, denoted by $K_{i}$, is, then, calculated as a weighted average of all the scores of the "leaf" attributes. The value of $K_{i}$ is between 1 and 5 and a high value indicates that we have stronger knowledge on that atomic element.

\subsection{Knowledge aggregation}

From (8), the risk index of the reduced-order PRA model is the sum of $n_{l}=\sum_{i=1}^{n_{O, R e d}} n_{S, R e d, i}$ risk index values $R_{R e d, l}$ from the corresponding elementary risk model, where each elementary risk model is further composed of MCS and BEs, as shown in (2):

$R_{R e d, l}=\sum_{k=1}^{n_{M C S, R e d, l}} \prod_{q \in M C S_{l, k}} P_{B E, q}$

In (9), $R_{R e d, l}$ is the risk index of the $l$-th reduced elementary risk model, where $l=1,2, \cdots, n_{l}$ and $n_{M C S, R e d, l}$ is the number of MCS in the $l$-th reduced elementary risk model.

Let $K_{B E, l, q}$ denote the strength of knowledge of the $q$-th BE in the reduced elementary risk, where $K_{B E, l, q} \in[1,5]$ and a large value of $K_{B E, l, q}$ indicates strong knowledge of BE. The $K_{B E, l, q} \mathrm{~s}$ are assessed using the procedures described in Sect. 2.2.

The next step is to aggregate the $K_{B E, l, q} \mathrm{~s}$ to assess the strength of knowledge of the whole risk assessment model. The aggregation should consider the difference in each atomic element's contribution to the total risk.

Different importance measures can be used to evaluate the contribution of the atomic elements with respect to the total risk. Since the elementary reduced-ordered risk model is constructed by the BEs through MCSs, the weights of the BEs are calculated based on Fussell-Vesely importance measures:

$$
W_{B E, l, q}=\frac{I_{B E, l, q}}{\sum_{q=1}^{n_{B E, l}} l_{B E, l, q}}
$$

where $I_{B E, i j}$ is the Fussell-Vesely importance measure of the corresponding $\mathrm{BE}$ in the elementary risk model $l$.

The strength of knowledge for the $l$-th elementary reduced order risk model, denoted by $K_{l}$, is calculated by:

$$
K_{l}=\sum_{q=1}^{n_{B E, l}} W_{B E, l, q} \cdot K_{B E, l, q}
$$

The importance of the elementary reduced-order model is evaluated by its contribution to the total risk:

$$
W_{l}=\frac{R_{R e d, l}}{\sum_{l=1}^{n_{l} R_{R e d, l}}}
$$

where $R_{R e d, l}$ is the risk index value of the $l$-th elementary reduced order model and is calculated by (9).

To calculate the total strength of knowledge $K_{R e d}$ of the reduced-order risk model, the knowledge indexes $K_{l} \mathrm{~s}$ of the reduced-order elementary risk models are further aggregated by considering their contributions:

$$
K_{\text {Red }}=\sum_{l=1}^{n_{l}} W_{l} K_{l}
$$

The index $K_{\text {Red }}$ is, then, used to represent the strength of knowledge of the entire PRA. Its value is between 1 and 5, and a high value indicates that we have strong knowledge in support of the PRA model and its risk outcomes.

\section{Case study}

\subsection{Problem description}

In this section, we apply the developed method to assess the strength of knowledge of NPPs PRAs. Two hazard groups, i.e., external hazards and internal events are considered in this case study.

External hazards refer to the undesired events originating from sources outside the NPPs such as: external flooding, external fires, seismic hazards, etc., [21]. In particular, external flooding is a naturally induced hazard that might be caused due to different reasons such as: tides, tsunamis, dam failures, snow melts, storm surges and etc., (see [22] for more examples). The choice of these initiating events to be a part of the external flooding risk assessment models is site-specific and some guidance should be provided for this purpose [23]. In general, for external flooding, the state of PRA practice is considered less mature than for internal events [24]. For example, the flood frequencies are obtained using statistical models and by extrapolating design basis flood levels to the fitted historical data (usually limited), which results in a very high uncertainty [24]. Moreover, for extreme floods, the probability of occurrence is very low but, on the other hand, the potential consequences can be catastrophic [22]. The low probability and the consequent lack of data experience introduces large uncertainties in the risk analysis of this type of events [22].

Internal events refer to the undesired events that originate within the NPPs itself, which cause initiating events that might lead to loss of important systems and might eventually result in core meltdown [1]. The internal events are mainly [25]: (i) different types of components, systems and structures failures, missiles and fires; (ii) safety systems operation and maintenance errors. These types of internal events can cause other initiating events such as turbine trip or Loss of Coolant Accidents (LOCAs). The 
risk assessment of internal events has been significantly developed and considered to have lower uncertainty compared to other hazard groups [1].

\subsection{Evaluation of hazard group strength of knowledge}

In this case study, we consider the risk analysis models of two hazard groups developed by Electricité de France (EDF) using Risk Spectrum Professional software [26]; [27]. The knowledge assessment framework developed in Section 2 is applied to evaluate the strength of knowledge of the risk models for both internal and external events. Technical reports were provided to the experts to support the knowledge assessment with the needed data and information. For simplification, we only present the case of the external events (specifically flooding). For internal events, we only show the results of the application.

\subsubsection{Reduced-order model construction}

Based on Eq. (4) with $\alpha=0.8$, we found that only one out of six operating states (NS/SG-normal shutdown with cooling using steam generator-NS/SG) is needed for the reduced-order model, which contributes to $86 \%$ of the total risk index. Similarly, based on Eq. (5) with $\beta=0.8$, only one out of ten scenarios (water levels) is needed for the reduced-order model, whose risk contribution is $98.7 \%$. Based on Eq. (7) with $\gamma=0.8$, the number of MCSs needed for the reduced-order model is 5 out of 3102 , and the risk contribution is $80.1 \%$.

Therefore, a reduced-order model is constructed based on the atomic elements in Table 3, as shown in Figure 4.

Table 3 Reduced-order model constituents

\begin{tabular}{|c|c|c|c|c|}
\hline $\begin{array}{c}\text { Operating } \\
\text { state }\end{array}$ & Scenarios & $\begin{array}{c}\text { Number } \\
\text { of MCS }\end{array}$ & $\begin{array}{c}\text { Number } \\
\text { of basic } \\
\text { events } \\
\text { BE }\end{array}$ & $\begin{array}{c}\text { Total risk } \\
\text { Contribution }\end{array}$ \\
\hline$N S / S G$ & $\begin{array}{c}\text { Water } \\
\text { level A }\end{array}$ & 5 & 10 & $0.86 \times 0.987 \times 0.801=$ \\
0.6799
\end{tabular}

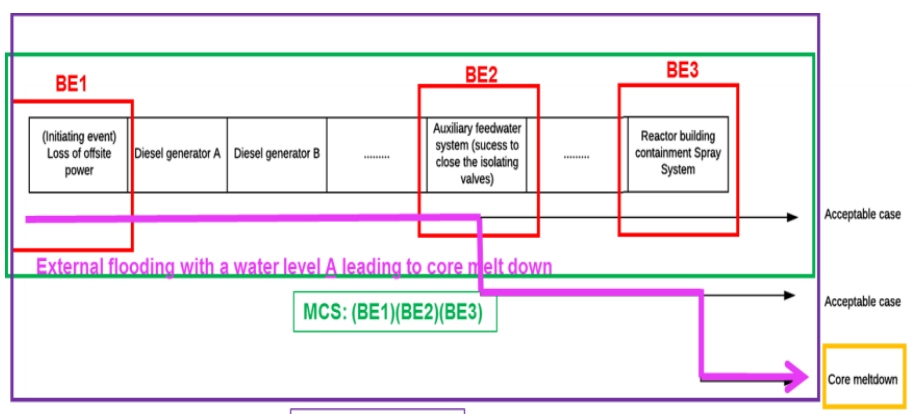

Operation state: $0 \mathrm{i}$

Figure 4 An illustration of the reduced-order model

\subsubsection{Strength of knowledge assessment}

After constructing the reduced-order model, the knowledge assessment framework in Section 2.2 has been applied on each of the atomic elements and, then, AHP is used to compare the overall model strength of knowledge. The strength of knowledge for external flooding turns out to be $K_{E F}=2.79$. The results of the knowledge assessment for both hazard groups are graphically illustrated in Figure 5. It can be seen from the Figure that the strength of knowledge on the internal events is higher than that on external flooding. In fact, these results confirm our expectations. In addition, most of the risk assigned to the external flooding is due to two basic events (failure to close the isolating valve in the auxiliary feedwater system and failure of the containment spray system), whose strength of knowledge is very weak.

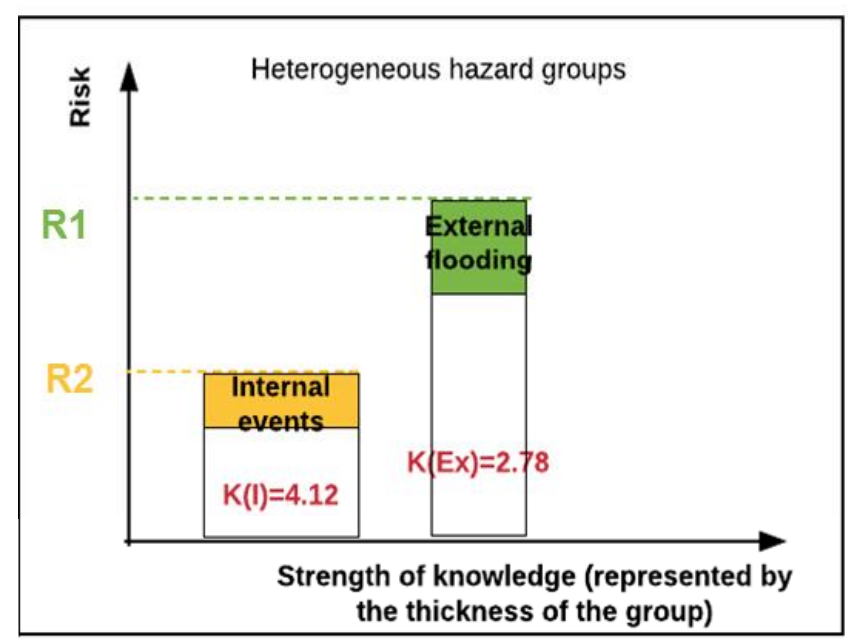

Figure 5 Representation of hazard groups levels of risk and strength of knowledge

\section{Conclusions}

An analytical hierarchy process-based framework has been proposed for assessing the strength of knowledge of PRA models. The framework is based on three main attributes (assumptions, data, and phenomenological understanding), which are further decomposed into sub-attributes and "leaf" attributes. A reduced-order PRA model is constructed, that reduces the number of atomic elements to be analyzed. The framework has been applied on two hazard groups in NPPs.

In the future, model uncertainty in the PRA model will be considered for a more comprehensive knowledge assessment. In addition, in the current framework, the weights of the attributes in the AHP were subjectively evaluated. Future investigations will be devoted on how to more objectively evaluate the weights.

\section{Reference}

[1] EPRI, "An Approach to Risk Aggregation for RisKInformed Decision-Making," Palo Alto, California, 2015 .

[2] T. Aven, "Practical implications of the new risk perspectives," Reliab. Eng. Syst. Saf., vol. 115, pp. 136-145, 2013.

[3] IAEA, "Technical Meeting on Managing Nuclear Safety Knowledge - Approaches and National Experiences," 2017.

[4] Flage and T. Aven, "Expressing and communicating uncertainty in relation to quantitative risk analysis," Reliab. Risk Anal. Theory Appl., vol. 2, no. 13, pp. 918, 2009.

[5] E. Zio, "An introduction to the basics of reliability and risk analysis. Series in Quality," Reliab. Eng. Stat., vol. 13, 2007.

[6] RELCON AB, "Theory Manual," 2005.

[7] R. Koch, The 80/20 principle: the secret to achieving more with less. Crown Business, 2011.

[8] C. Kelp, "Understanding phenomena," Synthese, vol. 192, no. 12, pp. 3799-3816, 2015.

[9] I. Boone et al., "NUSAP: a method to evaluate the 
quality of assumptions in quantitative microbial risk assessment," J. Risk Res., vol. 13, no. 3, pp. 337-352, 2010 .

[10] P. Kloprogge, J. P. Van der Sluijs, and A. C. Petersen, "A method for the analysis of assumptions in modelbased environmental assessments," Environ. Model. Softw., vol. 26, no. 3, pp. 289-301, 2011.

[11] P. A. Stirling, "On Science and Precaution in the Management of Technological Risk: Volume II-case studies," 1999.

[12] E. Laes, G. Meskens, and J. P. van der Sluijs, "On the contribution of external cost calculations to energy system governance: The case of a potential large-scale nuclear accident," Energy Policy, vol. 39, no. 9, pp. 5664-5673, 2011.

[13] J. P. Van Der Sluijs, M. Craye, S. Funtowicz, P. Kloprogge, J. Ravetz, and J. Risbey, "Combining Quantitative and Qualitative Measures of Uncertainty in Model-Based Environmental Assessment: The NUSAP System," Risk Anal., vol. 25, no. 2, pp. 481$492,2005$.

[14] E. Zio, "On the use of the analytic hierarchy process in the aggregation of expert judgments," Reliab. Eng. Syst. Saf., vol. 53, no. 2, pp. 127-138, 1996.

[15] M. Bergdahl, M. Ehling, E. Elvers, and E. Földesi, Handbook on Data Quality Assessment Methods and Tools. 2007.

[16] DAMA, "The six primary dimensions for data quality assessment: defining data quality dimensions," 2013.

[17] IAEA, Data Collection and Record Keeping for the Management of Nuclear Power Plant Ageing, no. 50. 1991.
[18] T. L. Saaty and L. G. Vargas, Models, methods, concepts \& applications of the analytic hierarchy process, vol. 175. Springer Science \& Business Media, 2012.

[19] M. Alexander, "Decision-Making using the Analytic Hierarchy Process (AHP) and SAS/ IML," United States Soc. Secur. Adm. Balt., pp. 1-12, 2012.

[20] T. L. Saaty, "Decision making with the analytic hierarchy process," Int. J. Serv. Sci., vol. 1, no. 1, p. 83, 2008 .

[21] IAEA, "Development and Application of Level 1 Probabilistic Safety Assessment for Nuclear Power Plants," 2010.

[22] IAEA, "External Events Excluding Earthquakes in the Design of Nuclear Power Plants," 2003.

[23] IAEA, "Meteorological and Hydrological Hazards in Site Evaluation for Nuclear Installations," 2009.

[24] EPRI, "Practical Guidance on the Use of Probabilistic Risk Assessment in Risk-Informed Applications with a Focus on the treatment of Uncertanity," Palo Alto, California, 2012.

[25] IAEA Safety Standards Series, "Deterministic Safety Analysis for Nuclear Power Plants," 2009.

[26] EDF, "Technical report: Qualitative Analysis for LOOP due to internal cause.".

[27] EDF, "Report technique: Approche Graduée - Analyse Probabiliste du Risque pour $1 \hat{a} €^{\mathrm{TM}}$ Inondation Externe : Quantification des Scénarios Accidentels," vol. 33, no. $1,2015$. 